\title{
A Flatness Property for Filtered $\mathcal{D}$-modules
}

\author{
By
}

\author{
Francisco J. CAStro-JimÉnEZ* and Michel Granger**
}

\begin{abstract}
Let $\mathcal{M}$ be a coherent module over the ring $\mathcal{D}_{X}$ of linear differential operators on an analytic manifold $X$ and let $Z_{1}, \ldots, Z_{k}$ be $k$ germs of transverse hypersurfaces at a point $x \in X$. The Malgrange-Kashiwara V-filtrations along these hypersurfaces, associated with a given presentation of the germ of $\mathcal{M}$ at $x$, give rise to a multifiltration $U_{\bullet}(\mathcal{M})$ of $\mathcal{M}_{x}$ as in Sabbah's paper [9] and to an analytic standard fan in a way similar to [3]. We prove here that this standard fan is adapted to the multifiltration, in the sense of C. Sabbah. This result completes the proof of the existence of an adapted fan in [9], for which the use of [8] is not possible.
\end{abstract}

\section{§1. Introduction}

Let us consider a coherent module $\mathcal{M}$ over the ring $\mathcal{D}_{X}$ of differential operators on an analytic manifold $X$. For any smooth hypersurface $Z$ of $X$, Malgrange and Kashiwara defined a filtration along $Z$ for $\mathcal{D}_{X}$ and the notion of a good filtration for $\mathcal{M}$. Given a set of transverse smooth hypersurfaces $Z_{1}, \ldots, Z_{k}$, Sabbah considered in [9] multifiltrations of $\mathcal{M}$ indexed by $k$-uples of integers. To be precise he dealt with linear combinations over $\mathbf{Q}^{+}$of the filtrations $V^{(j)}$ along each hypersurface $Z_{j}$ and with refinements $V^{\Gamma}$ of the original multifiltration associated with each rational polyhedral simplicial cone in the positive quadrant of $\left(\mathbf{Q}^{k}\right)^{\star}$. The original multifiltration is the one which corresponds to the case $\Gamma=\mathbf{N}^{k}$.

Communicated by M. Kashiwara. Received July 25, 2005. Revised November 21, 2005. 2000 Mathematics Subject Classification(s): Primary 32C38, 13C11, 14Q99, 13P99, $68 \mathrm{~W} 30$.

Partially supported by MTM2004-01165 and Picasso-A.I. HF2004-0117.

* University of Sevilla, Spain.

e-mail: castro@us.es

** University of Angers, France.

e-mail: granger@univ-angers.fr

(C) 2007 Research Institute for Mathematical Sciences, Kyoto University. All rights reserved. 
The aim of this paper is to clarify the flatness properties which appear in [9], namely to prove the existence of a fan $\mathcal{E}$, such that for any cone $\Gamma$ in this fan, the Rees module of the filtration $V^{\Gamma}$ is flat over the affine ring $A_{\Gamma}$ of the toric blowing-up of $\mathbf{C}^{k}$ associated with the fan $\mathcal{E}$. Such a fan is called an adapted fan in [9]. The reason for this clarification is that the proof in [9] depends on the appendix [8] in which the main tool is a division theorem in Rees rings of differential operators which is not correct as stated. Indeed, the infinite process that its proof suggests, would require monomials of unbounded degrees in the differential variables of the ring of operators.

One of the main consequences of the existence of an adapted fan as developed in [9] is that we thus complete the proof of the existence of non trivial functional equations of Bernstein-Sato type for a $k$-uple of functions, following the argument of Sabbah in [9]. It should be emphasized here that this problem involves a $\mathcal{D}_{X \times \mathbf{C}^{k}}$-module naturally associated with a $k$-uple of functions on $X$, and that in this situation a multifiltration, along the transverse hypersurfaces $t_{j}=0$ due to the factor $\mathbf{C}^{k}$, appears in a natural way.

We must note here that the proof of the existence of Bernstein-Sato equations has already been completed in Bahloul's paper [4], by a different method which avoids the reference to a flatness property involved in the notion of an adapted fan. Bahloul uses instead the analytic standard fan as defined in [3], and described also in [5]. It is therefore not completely surprising that the adapted fan wanted in [9] turns out in fact to be the analytic standard fan. This emphasizes the interest of Bahloul's proof which also has the advantage of being constructive. This constructiveness is valuable in an algebraic setting also. In both proofs, Bahloul's and Sabbah's, the latter which is completed by this paper, the main step is the proof of the goodness of the so-called saturated filtration. The details are given in Section 2.3.

The starting point of the proof of our main theorem (Theorem 4.3) is a criterion of flatness by M. Herrmann and U. Orbanz (see [7]), for graded modules over graded rings, where the grading is indexed by an arbitrary commutative group. In the statement of this result there is no reference to any finiteness property. In our case the indexing group will be $\mathbf{Z}^{k}$, and the ring a conical subring of the ring of Laurent polynomials in $k$ variables with the obvious multigrading by monomials. The main ingredient of the proof of Theorem 4.3 is then the existence of a simultaneous $L$-standard basis of a submodule $\mathcal{N} \subset \mathcal{D}^{r}$ for all $L$ in a cone of the analytic standard fan of $\mathcal{N}$ (see [3]).

The plan of the paper is as follows. In Section 2, we recall with more details what $V$-multifiltrations, and Rees modules are, and the refinements of 
these multifiltrations with respect to a rational polyhedral simplicial cone. We end this Section by defining the fiber at zero of these Rees modules seen as modules over the ring of an affine toric blowing up. We aim to prove that this is the fiber of a flat deformation if the cone is taken in the analytic standard fan.

In Section 3 we recall the notion of an analytic standard fan as developed in [3], and we sketch the easy generalization which we need from the cyclic case treated in [3] to the general case.

In Section 4 we finally prove that the analytic standard fan is an adapted fan in the sense of [9].

\section{§2. Multifiltrations, Rees Rings and Rees Modules}

Let us denote by $\mathcal{O}=\mathbf{C}\left\{x_{1}, \ldots, x_{n}\right\}$ the complex convergent power series ring and by $\mathcal{D}$ the ring of germs of linear differential operators with holomorphic coefficients (i.e. $\mathcal{D}=\mathcal{O}[\partial]=\mathcal{O}\left[\partial_{1}, \ldots, \partial_{n}\right]$ where $\partial_{i}$ is the partial derivative with respect to $x_{i}$ and the product in $\mathcal{D}$ is defined by the Leibnitz's rule: $\partial_{i} a=a \partial_{i}+\partial_{i}(a)$, for each $\left.a \in \mathcal{O}\right)$.

An operator $P$ in $\mathcal{D}$ can be written as

$$
P=\sum_{\beta \in \mathbf{N}^{n}} p_{\beta}(x) \partial^{\beta}=\sum_{\alpha, \beta \in \mathbf{N}^{n}} p_{\alpha \beta} x^{\alpha} \partial^{\beta}
$$

where the first sum is finite, $\alpha=\left(\alpha_{1}, \ldots, \alpha_{n}\right), \beta=\left(\beta_{1}, \ldots, \beta_{n}\right), x^{\alpha}=$ $x_{1}^{\alpha_{1}} \cdots x_{n}^{\alpha_{n}}, \partial^{\beta}=\partial_{1}^{\beta_{1}} \cdots \partial_{n}^{\beta_{n}}, p_{\beta}(x) \in \mathcal{O}$ and $p_{\alpha \beta} \in \mathbf{C}$.

For each $i=1, \ldots, n$, let us remember that the $V$-filtration on $\mathcal{D}$, with respect to the hypersurface $x_{i}=0$, was defined by Malgrange and Kashiwara as:

$$
V_{\ell}^{(i)}(\mathcal{D})=V_{\ell}^{(i)}=\left\{P=\sum_{\alpha, \beta \in \mathbf{N}^{n}} p_{\alpha \beta} x^{\alpha} \partial^{\beta} \in \mathcal{D} \mid \beta_{i}-\alpha_{i} \leq \ell\right\}
$$

for each $\ell \in \mathbf{Z}$. The family $\left(V_{\ell}^{(i)}\right)_{\ell \in \mathbf{Z}}$ is an increasing exhaustive filtration on the ring $\mathcal{D}$. For $i=1$, the associated graded ring

$$
\operatorname{gr}^{(1)}(\mathcal{D})=\bigoplus_{\ell} \frac{V_{\ell}^{(1)}}{V_{\ell-1}^{(1)}}
$$

is isomorphic to the ring $\mathbf{C}\left\{x_{2}, \ldots, x_{n}\right\}\left[x_{1}, \partial_{1}, \partial_{2}, \ldots, \partial_{n}\right]$, graded by the socalled $V^{(1)}$-graduation, where the homogeneous elements of $V^{(1)}$-degree $\ell$ are

$$
\sum_{\alpha, \beta \in \mathbf{N}^{n} ; \beta_{1}-\alpha_{1}=\ell} p_{\alpha \beta} x^{\alpha} \partial^{\beta} .
$$


We have similar descriptions for $i=2, \ldots, k$.

We can also consider a $V^{(i)}$-filtration on the free module $\mathcal{D}^{r}$ just by defining $V_{\ell}^{(i)}\left(\mathcal{D}^{r}\right)=\left(V_{\ell}^{(i)} \mathcal{D}\right)^{r}$ and more generally, for any vector $\underline{m}=\left(m_{1}, \ldots, m_{r}\right) \in$ $\mathbf{Z}^{r}$, one can define the shifted filtration $V^{(i)}[\underline{m}]_{\ell}\left(\mathcal{D}^{r}\right)=\oplus_{j=1}^{r} V_{\ell-m_{j}}^{(i)} \mathcal{D}$. Such a filtered module is called a $V^{(i)}$-filtered free module. All the $\mathcal{D}$-modules considered will be left modules.

Definition 2.1. $\quad$ Let $\mathcal{M}$ be a $\mathcal{D}$-module. We say that a filtration $U^{(i)} \times$ $(\mathcal{M})$ indexed by $\mathbf{Z}$ is a good $V^{(i)}$-filtration if there exists a presentation $\mathcal{M}=\frac{\mathcal{D}^{r}}{\mathcal{N}}$ as a quotient by a left submodule $\mathcal{N}$ of $\mathcal{D}^{r}$, and a weight vector $\underline{m}$ such that $U_{\ell}^{(i)}(\mathcal{M})=\pi\left(V^{(i)}[\underline{m}]_{\ell}\left(\mathcal{D}^{r}\right)\right)$ where $\pi$ is the projection $\mathcal{D}^{r} \rightarrow \mathcal{M}$.

\section{§2.1. $\quad V$-multifiltrations and $V^{\Gamma}$-multifiltrations}

Let us fix an integer $k$ such that $1 \leq k \leq n$.

For each $s=\left(s_{1}, \ldots, s_{k}\right) \in \mathbf{Z}^{k}$, we shall denote $V_{s}(\mathcal{D})=\bigcap_{i=1}^{k} V_{s_{i}}^{(i)}(\mathcal{D})$. The family $\left\{V_{s}(\mathcal{D})\right\}_{s \in \mathbf{Z}^{k}}$ defines a multi-filtration on the ring $\mathcal{D}$. To simplify we shall say that $V_{\bullet}(\mathcal{D})$ is a $k$-filtration on $\mathcal{D}$ or even, if no confusion is possible, a filtration on $\mathcal{D}$.

Let us consider a rational simplicial cone $\Gamma$ in the first quadrant of the dual space $\left(\mathbf{Q}^{k}\right)^{*}=\operatorname{Hom}_{\mathbf{Q}}\left(\mathbf{Q}^{k}, \mathbf{Q}\right)$. We denote by $\check{\Gamma}$ the dual cone of $\Gamma$, i.e.

$$
\check{\Gamma}=\left\{a \in \mathbf{Q}^{k} \mid \gamma(a) \geq 0, \forall \gamma \in \Gamma\right\} .
$$

We associate with such a cone $\Gamma$ the affine variety, denoted by $S_{\Gamma}$, with coordinate ring equal to $\mathbf{C}\left[\check{\Gamma} \cap \mathbf{Z}^{k}\right]$. We will denote $A_{\Gamma}=\mathbf{C}\left[\check{\Gamma} \cap \mathbf{Z}^{k}\right]$ and $A=\mathbf{C}\left[\mathbf{N}^{k}\right]$. We denote by $\mathcal{L}(\Gamma)$ the set of primitive elements in the 1-skeleton of $\Gamma$.

The multifiltration $V^{\Gamma}$ on $\mathcal{D}$ is defined as follows: For each $s \in \mathbf{Z}^{k}$ we define

$$
V_{s}^{\Gamma}(\mathcal{D})=\sum_{\sigma \in \mathbf{Z}^{k} \mid L(\sigma) \leq L(s) ; \forall L \in \mathcal{L}(\Gamma)} V_{\sigma}(\mathcal{D}) .
$$

Notice that the sum is indexed by $\sigma \in s-\check{\Gamma}$ and that we have the inclusion $V_{s}(\mathcal{D}) \subset V_{s}^{\Gamma}(\mathcal{D})$. The family $V_{\bullet}^{\Gamma}(\mathcal{D})$ is a multifiltration of the ring $\mathcal{D}$, indexed by $s \in \mathbf{Z}^{k}$. This means that:

$$
V_{s}^{\Gamma}(\mathcal{D}) . V_{s^{\prime}}^{\Gamma}(\mathcal{D}) \subset V_{s+s^{\prime}}^{\Gamma}(\mathcal{D}) \text { and } \bigcup V_{s}^{\Gamma}(\mathcal{D})=\mathcal{D} .
$$

We may define, in a similar way to the case of one filtration, the notion of a free multi-filtered module and that of a good multifiltration of a finitely generated $\mathcal{D}$-module $\mathcal{M}$. For that purpose we chose a shift multivector $\underline{n}=$ $\left(n^{(1)}, \ldots, n^{(r)}\right) \in\left(\mathbf{Z}^{k}\right)^{r}$ called also a shift matrix, with columns $n^{(i)} \in \mathbf{Z}^{k}$, and a presentation $\mathcal{M}=\frac{\mathcal{D}^{r}}{\mathcal{N}}$ of $\mathcal{M}$. 
Definition 2.2. The multifiltered free module associated with $\underline{n}$ is the module $\mathcal{D}^{r}$ endowed with the multifiltration indexed by $s \in \mathbf{Z}^{k}$, and defined as:

$$
V[\underline{n}]_{s}\left(\mathcal{D}^{r}\right)=\bigoplus_{i=1}^{r} V_{s-n^{(i)}}(\mathcal{D})
$$

Definition 2.3. A good multifiltration of $\mathcal{M}$, is a filtration indexed by $s \in \mathbf{Z}^{k}$, of the type

$$
U_{s}(\mathcal{M})=\pi\left(V[\underline{n}]_{s}\left(\mathcal{D}^{r}\right)\right)=\frac{V[\underline{n}]_{s}\left(\mathcal{D}^{r}\right)+\mathcal{N}}{\mathcal{N}}
$$

for some presentation $\pi: \mathcal{D}^{r} \rightarrow \mathcal{M}$ of $\mathcal{M}$

In the obvious sense these multifiltrations are compatible with the multifiltered structure on the ring $\mathcal{D}$. Remark that the $i$-th generator $e_{i}$ of $\mathcal{D}^{r}$ is then of multidegree $n^{(i)} \in \mathbf{Z}^{k}$. We may also endow $\mathcal{N}$ with the induced filtration $U_{s}(\mathcal{N})=V[\underline{n}]_{s}\left(\mathcal{D}^{r}\right) \cap \mathcal{N}$, so that we also have

$$
U_{s}(\mathcal{M})=\frac{V[\underline{n}]_{s}\left(\mathcal{D}^{r}\right)}{U_{s}(\mathcal{N})} .
$$

We may observe that the multifiltration on the free module $\mathcal{D}^{r}$ is defined as the intersection of the $V^{(i)}$-filtration with respect to the row vectors of $\underline{n}$, $\underline{n}_{i}=\left(n_{i}^{(1)}, \ldots, n_{i}^{(r)}\right) \in \mathbf{Z}^{r}$, that is

$$
V[\underline{n}]_{s}\left(\mathcal{D}^{r}\right)=V^{(1)}\left[\underline{n}_{1}\right]_{s_{1}}\left(\mathcal{D}^{r}\right) \cap \cdots \cap V^{(k)}\left[\underline{n}_{k}\right]_{s_{k}}\left(\mathcal{D}^{r}\right),
$$

but the analogue for $\mathcal{M}$, with respect to the good $V^{(i)}$-filtrations as defined in Definition 2.1 is not true since the inclusion:

$$
\begin{aligned}
U_{s}(\mathcal{M})= & \frac{V^{(1)}\left[\underline{n}_{1}\right]_{s_{1}}\left(\mathcal{D}^{r}\right) \cap \cdots \cap V^{(k)}\left[\underline{n}_{k}\right]_{s_{k}}\left(\mathcal{D}^{r}\right)+\mathcal{N}}{\mathcal{N}} \\
& \subset \bigcap_{i=1}^{k} \frac{V^{(i)}\left[\underline{n}_{i}\right]_{s_{i}}\left(\mathcal{D}^{r}\right)+\mathcal{N}}{\mathcal{N}}=\bigcap_{i=1}^{k} U_{s_{i}}^{(i)}(\mathcal{M})
\end{aligned}
$$

may be strict.

For each good filtration there is an associated $\Gamma$-filtration compatible with the multifiltration $V_{\bullet}^{\Gamma}(\mathcal{D})$ on $\mathcal{D}$ :

For each $s \in \mathbf{Z}^{k}$ let us consider

$$
U_{s}^{\Gamma}(\mathcal{M})=\sum_{\sigma \in \mathbf{Z}^{k} \mid L(\sigma) \leq L(s) ; \forall L \in \mathcal{L}(\Gamma)} U_{\sigma}(\mathcal{M}) .
$$


The multifiltration $U_{\bullet}^{\Gamma}(\mathcal{M})$ is a good filtration with respect to $V_{\bullet}^{\Gamma}(\mathcal{D})$.

The goodness means here that we can verify, with the presentation of $\mathcal{M}$ as above, that $U_{s}^{\Gamma}(\mathcal{M})=\pi\left(V[\underline{n}]_{s}^{\Gamma}\left(\mathcal{D}^{r}\right)\right)$, is still the quotient of a filtration on the free module $\mathcal{D}^{r}$ which is a direct sum of convenient shifts of the filtration $V_{\bullet}^{\Gamma}$ on the ring $\mathcal{D}$. More precisely the involved filtration $V[\underline{n}]_{\bullet}^{\Gamma}$ is defined by

$$
V[\underline{n}]_{s}^{\Gamma}\left(\mathcal{D}^{r}\right)=\sum_{\sigma \in \mathbf{Z}^{k} \mid L(\sigma) \leq L(s) ; \forall L \in \mathcal{L}(\Gamma)} V[\underline{n}]_{\sigma}\left(\mathcal{D}^{r}\right)\left(=\bigoplus_{i=1}^{r}\left(V_{s-n^{(i)}}^{\Gamma}(\mathcal{D})\right)\right.
$$

and it remains to be remarked that:

$$
\begin{aligned}
U_{s}^{\Gamma}(\mathcal{M}) & =\sum_{\sigma \in \mathbf{Z}^{k} \mid L(\sigma) \leq L(s) ; \forall L \in \mathcal{L}(\Gamma)} U_{\sigma}(\mathcal{M}) \\
& =\sum_{\sigma \in \mathbf{Z}^{k} \mid L(\sigma) \leq L(s) ; \forall L \in \mathcal{L}(\Gamma)} \frac{V[\underline{n}]_{\sigma}\left(\mathcal{D}^{r}\right)+\mathcal{N}}{\mathcal{N}}=\frac{V[\underline{n}]_{s}^{\Gamma}\left(\mathcal{D}^{r}\right)+\mathcal{N}}{\mathcal{N}}
\end{aligned}
$$

so that the goodness of the filtration $U_{\bullet}(\mathcal{M})$ implies the goodness of $U_{\bullet}^{\Gamma}(\mathcal{M})$.

\section{§2.2. $\quad$ Rees rings and Rees modules}

2.2.1. Definition of Rees rings $\mathcal{R}_{V}(\mathcal{D}), \mathcal{R}^{\Gamma}(\mathcal{D})$ and of related Rees modules The Rees ring associated with the $V$-multifiltration on $\mathcal{D}$ is defined by:

$$
\mathcal{R}_{V}(\mathcal{D})=\bigoplus_{s \in \mathbf{Z}^{k}} V_{s}(\mathcal{D}) u^{s}
$$

where $u=\left(u_{1}, \ldots, u_{k}\right)$ are variables and the product in the Rees ring is induced by the natural product in the Laurent polynomial ring $\mathcal{D}\left[u^{ \pm 1}\right]=$ $\mathcal{D}\left[u_{1}, u_{1}^{-1}, \ldots, u_{k}, u_{k}^{-1}\right]$.

By definition the Rees ring $\mathcal{R}_{V}(\mathcal{D})$ is a graded $\mathbf{C}$-algebra with values group $\mathbf{Z}^{k}$, whose homogeneous elements are $P u^{s}$ for $P \in V_{s}(\mathcal{D})$ and $s \in \mathbf{Z}^{k}$.

Similarly given a $\mathcal{D}$-module $\mathcal{M}=\mathcal{D}^{r} / \mathcal{N}$ and a shift matrix $\underline{n}$, we define from the good filtrations $V[\underline{n}]_{s}\left(\mathcal{D}^{r}\right)$, and $U_{s}(\mathcal{M})$, the Rees modules

$$
\begin{aligned}
\mathcal{R}_{V[\underline{n}]}\left(\mathcal{D}^{r}\right) & =\oplus_{s \in \mathbf{Z}^{k}} V[\underline{n}]_{s}\left(\mathcal{D}^{r}\right) u^{s} \\
\mathcal{R}_{U}(\mathcal{M}) & =\bigoplus_{s \in \mathbf{Z}^{k}} U_{s}(\mathcal{M}) u^{s} .
\end{aligned}
$$

Both have a natural structure of mutigraded left $\mathcal{R}_{V}(\mathcal{D})$-module and we shall consider $\mathcal{R}_{U}(\mathcal{M})$ as a sub-group of $\mathcal{M}\left[u^{ \pm 1}\right]$, the Laurent polynomials with coefficients in $\mathcal{M}$. 
As $\mathcal{N}$ is a left submodule of $\mathcal{D}^{r}$, we can also consider on $\mathcal{N}$ the induced $V$-filtration:

$$
V_{s}(\mathcal{N}):=\mathcal{N} \cap V_{s}[\underline{n}]\left(\mathcal{D}^{r}\right)
$$

for each $s \in \mathbf{Z}^{k}$ as defined before (see 2.1). The abelian group

$$
\mathcal{R}_{V}(\mathcal{N}):=\bigoplus_{s \in \mathbf{Z}^{k}} V_{s}(\mathcal{N}) u^{s}
$$

is in fact an homogeneous submodule of $\mathcal{R}_{V[\underline{\underline{n}}]}\left(\mathcal{D}^{r}\right)$. The $\mathcal{R}_{V}(\mathcal{D})$-module $\mathcal{R}_{U}(\mathcal{M})$ is naturally isomorphic to the quotient $\mathcal{R}_{V[\underline{n}]}\left(\mathcal{D}^{r}\right) / \mathcal{R}_{V}(\mathcal{N})$.

Definition 2.4. We call the module $\mathcal{R}_{U}(\mathcal{M})$ the Rees module associated with the filtration $U_{\bullet}(\mathcal{M})$.

Let us now consider the filtration $V_{\bullet}^{\Gamma}(\mathcal{D})$ as in 2.1. Then the Rees ring associated with this filtration is defined in a similar way as

$$
\mathcal{R}^{\Gamma}(\mathcal{D})=\bigoplus_{s \in \mathbf{Z}^{k}} V_{s}^{\Gamma}(\mathcal{D}) u^{s} \subset \mathcal{D}\left[u^{ \pm 1}\right]
$$

the product being induced by the one of $\mathcal{D}\left[u^{ \pm 1}\right]$.

If $\mathcal{M}=\frac{\mathcal{D}^{r}}{\mathcal{N}}$ is a finitely generated $\mathcal{D}$-module and $U \cdot(\mathcal{M})$ is a good filtration on $\mathcal{M}$ with respect to $V_{\bullet}(\mathcal{D})$ then, for any cone $\Gamma$ as in 2.1, the abelian group

$$
\mathcal{R}^{\Gamma}(\mathcal{M})=\bigoplus_{s \in \mathbf{Z}^{k}} U_{s}^{\Gamma}(\mathcal{M}) u^{s} \subset \mathcal{M}\left[u^{ \pm 1}\right]
$$

is a left graded $\mathcal{R}^{\Gamma}(\mathcal{D})$-module.

Definition 2.5. We call the module $\mathcal{R}^{\Gamma}(\mathcal{M})$ the Rees module associated with the filtration $U_{\bullet}(\mathcal{M})$ and the cone $\Gamma$.

The fact that a filtration is good in the sense of previous Section 2.1 is then equivalent to the fact that the Rees module of $\mathcal{M}$ is finitely generated over $\mathcal{R}_{V}(\mathcal{D})$. Similarly the goodness of $U_{\bullet}^{\Gamma}(\mathcal{M})$ is equivalent to the fact that the Rees module $\mathcal{R}^{\Gamma}(\mathcal{M})$ as defined above, is finitely generated over $\mathcal{R}^{\Gamma}(\mathcal{D})$. Since $\mathcal{R}^{\Gamma} \mathcal{D}$ is a Noetherian ring, this implies in particular an Artin type property: If $\mathcal{M}^{\prime} \subset \mathcal{M}$ is a submodule of a $\mathcal{D}$-module $\mathcal{M}$ endowed with a good filtration $U_{\bullet}(\mathcal{M})$ then the induced filtration on $\mathcal{M}^{\prime}$, namely $U_{\bullet}^{\Gamma}(\mathcal{M}) \cap \mathcal{M}^{\prime}$ is good.

The ring $\mathcal{R}^{\Gamma}(\mathcal{D})$ contains as a subring the $\mathbf{C}$-algebra $A_{\Gamma}=\mathbf{C}\left[\check{\Gamma} \cap \mathbf{Z}^{k}\right] \subset$ $\mathbf{C}\left[u, u^{-1}\right]$, all these rings being included in $\mathcal{D}\left[u, u^{-1}\right]$.

Theorem 2.6. There is a fan $\mathcal{E}$ in $\left(\mathbf{Q}^{k}\right)_{+}^{*}$ such that for each cone $\Gamma \in \mathcal{E}$ the Rees module $\mathcal{R}^{\Gamma}(\mathcal{M})$ is flat over $A_{\Gamma}$. 
We will prove a more precise form of this result we in Section 4, see Theorem 4.3, after having recalled in Section 3 the notion of an analytic standard fan.

2.2.2. The fiber at $0 \quad$ Let us denote by $\mathfrak{m}$ the ideal of $\mathcal{R}_{V}(\mathcal{D})$ generated by $\left(u_{1}, \ldots, u_{k}\right)$. This is a two-sided ideal since it is generated by central elements. The fiber at zero of $\mathcal{R}_{V}(\mathcal{D})$ (resp. of $\mathcal{R}_{V[\underline{n}]}\left(\mathcal{D}^{r}\right)$ ) is by definition the quotient ring (resp. the quotient module),

$$
\frac{\mathcal{R}_{V}(\mathcal{D})}{\mathfrak{m}}\left(\operatorname{resp} \cdot \frac{\mathcal{R}_{V[\underline{n}]}\left(\mathcal{D}^{r}\right)}{\mathfrak{m} \cdot \mathcal{R}_{V[\underline{n}]}\left(\mathcal{D}^{r}\right)}\right) .
$$

More generally the fiber at zero of $\mathcal{R}_{U}(\mathcal{M})=\frac{\mathcal{R}_{V[n]}\left(\mathcal{D}^{r}\right)}{\mathcal{R}_{V}(\mathcal{N})}$ is the quotient

$$
\frac{\mathcal{R}_{U}(\mathcal{M})}{\mathfrak{m} \mathcal{R}_{U}(\mathcal{M})}
$$

which is naturally isomorphic to the quotient

$$
\frac{\mathcal{R}_{V[\underline{n}]}\left(\mathcal{D}^{r}\right)}{\mathcal{R}_{V}(\mathcal{N})+\mathfrak{m} \cdot \mathcal{R}_{V[\underline{n}]}\left(\mathcal{D}^{r}\right)} .
$$

Notice that the fiber at 0 of the module $\mathcal{R}_{U}(\mathcal{M})$ can be zero for a non-zero $\mathcal{R}_{U}(\mathcal{M})$, as shown in the following example. Let us denote by $I$ the principal ideal of $\mathcal{D}$ generated by the differential operator $P=1+x_{1}^{2} \partial_{1}$ and let us suppose $k \geq 1$. Then $\mathcal{R}_{V}(I)$ is the principal ideal of $\mathcal{R}_{V}(\mathcal{D})$ generated by $P u_{1}^{0}=1+\left(x_{1}^{2} \partial_{1} u_{1}^{-1}\right) u_{1}$ and the fiber at zero of $\mathcal{R}_{V}(\mathcal{D} / I)$ is then zero since $\mathcal{R}_{V}(I)+\mathfrak{m} \cdot \mathcal{R}_{V}(\mathcal{D})$ contains $P-\left(x_{1}^{2} \partial_{1} u_{1}^{-1}\right) u_{1}=1$, so that it is equal to $\mathcal{R}_{V}(\mathcal{D})$.

Similarly for any $k$-dimensional cone $\Gamma$ the $\mathbf{C}$-algebra $A_{\Gamma}=\mathbf{C}\left[\check{\Gamma} \cap \mathbf{Z}^{k}\right]$ has a maximal ideal

$$
\mathfrak{m}^{\Gamma}=\mathbf{C}\left[\check{\Gamma} \cap \mathbf{Z}^{k} \backslash\{0\}\right]
$$

because $\check{\Gamma}$ is strictly convex. Then we define the fiber at zero of the Rees module $\mathcal{R}^{\Gamma}(\mathcal{M})$ as $\frac{\mathcal{R}^{\Gamma}(\mathcal{M})}{\mathfrak{m}^{\Gamma} \mathcal{R}^{\Gamma}(\mathcal{M})}$. As is explained in [9], this fiber is a module over the ring $\frac{\mathcal{R}^{\Gamma}(\mathcal{D})}{\mathfrak{m}^{\Gamma} \mathcal{R}^{\Gamma}(\mathcal{D})} \simeq g r^{\Gamma} \mathcal{D}$

2.2.3. Description of Rees rings $\mathcal{R}_{V}(\mathcal{D})$ and $\mathcal{R}^{\Gamma}(\mathcal{D}) \quad$ It is useful to describe the Rees ring $\mathcal{R}_{V}=\mathcal{R}_{V}(\mathcal{D})$ (resp. $\mathcal{R}^{\Gamma}(\mathcal{D})$ ) as subrings of the ring of relative differential operators $\mathcal{D}_{\mathbf{C}^{n} \times \mathbf{C}^{k} / \mathbf{C}^{k}}\left(\operatorname{resp} \mathcal{D}_{\mathbf{C}^{n} \times S_{\Gamma} / S_{\Gamma}}\right)$. This is nothing but an explicit version of the interpretation of a Rees ring as a ring of relative differential operators on the deformation of $Y \times 0_{\Gamma}$ to its normal cone in $\mathbf{C}^{n} \times S_{\Gamma}$, see [9]. We denote by $S_{\Gamma}$ the algebraic variety associated to the ring $A_{\Gamma}$. 
Let us define first

$$
\mathcal{A}=\mathbf{C}\left\{X^{\prime} U, X^{\prime \prime}\right\}\left[X^{\prime}, U, \Delta\right]=\mathbf{C}\left\{X_{1} U_{1}, \ldots, X_{k} U_{k}, X_{k+1}, \ldots, X_{n}\right\}\left[X^{\prime}, U, \Delta\right]
$$

where $U=\left(U_{1}, \ldots, U_{k}\right), X^{\prime}=\left(X_{1}, \ldots, X_{k}\right), X^{\prime \prime}=\left(X_{k+1}, \ldots, X_{n}\right), X=$ $\left(X_{1}, \ldots, X_{n}\right), \Delta=\left(\Delta_{1}, \ldots, \Delta_{n}\right)$ are new variables satisfying the following relations, for $i=1, \ldots, n$ :

$$
\Delta_{i} X_{i}=X_{i} \Delta_{i}+1
$$

the other relations being trivial.

The ring $\mathcal{A}$ is graded with $\mathbf{Z}^{k}$ as a values group (we will say that $\mathcal{A}$ is a $\mathbf{Z}^{k}$-graded ring), namely we have

$$
\mathcal{A}=\bigoplus_{s \in \mathbf{Z}^{k}} \mathcal{A}_{s}
$$

where $\mathcal{A}_{s}$ is the set

$$
\mathcal{A}_{s}=\left\{\begin{array}{c}
\sum_{\alpha, \beta \in \mathbf{N}^{n} ; \sigma \in \mathbf{N}^{k}} f_{\alpha \beta \sigma} X^{\alpha} \Delta^{\beta} U^{\sigma} \in \mathcal{A} \mid \begin{array}{c}
f_{\alpha \beta \sigma} \in \mathbf{C}\left\{X^{\prime} U, X^{\prime \prime}\right\} \\
\sigma_{i}+\beta_{i}-\alpha_{i}=s_{i} ; i=1, \ldots, k
\end{array}
\end{array} .\right.
$$

Proposition 2.7. $\quad$ There is an isomorphism of $\mathbf{Z}^{k}$-graded rings

$$
i=i_{V}: \mathcal{R}_{V}(\mathcal{D}) \rightarrow \mathcal{A}
$$

defined by:

- $i\left(u_{j}\right)=U_{j}$ for $j=1, \ldots, k$.

- $i\left(x_{j} u_{j}^{-1}\right)=X_{j}$ for $j=1, \ldots, k \quad$ and $\quad i\left(x_{j}\right)=X_{j}$ for $j=k+1, \ldots, n$.

- $i\left(\partial_{j} u_{j}\right)=\Delta_{j}$ for $j=1, \ldots, k \quad$ and $\quad i\left(\partial_{j}\right)=\Delta_{j}$ for $j=k+1, \ldots, n$.

Proof. It is clear that $i$ is injective and that, by the formula $i\left(x^{\alpha} \partial^{\beta} u^{s}\right)=$ $X^{\alpha} \Delta^{\beta}\left(\Pi_{i=1}^{k} U_{i}^{s_{i}+\alpha_{i}-\beta_{i}}\right), i\left(V_{s}(\mathcal{D}) u^{s}\right)=\mathcal{A}_{s}$ for all $s \in \mathbf{Z}^{k}$.

Let us give now the same description for the ring $\mathcal{R}^{\Gamma}(\mathcal{D})$. We shall do it in the only case of interest for us, when the cone $\Gamma$ is basic, which means that if it is defined by $k$ independent linear forms $\left\{L_{1}, \ldots, L_{k}\right\}$ generating the lattice $\mathbf{Z}^{k}$.

In this situation we are going to make a change of variables in order to write $A_{\Gamma}$ as a polynomial ring. 
For any $i$ we can write $L_{i}=\left(\ell_{i 1}, \ldots, \ell_{i k}\right)$ and we can suppose by a suitable ordering of the linear forms $L_{i}$ that the determinant of the matrix $\mathcal{L}=\left(\ell_{i j}\right)$ equals 1 .

Let us write $\mathcal{L}^{\prime}$ for the inverse matrix of $\mathcal{L}$, then the columns $\left\{C_{1}, \ldots, C_{k}\right\}$ of $\mathcal{L}^{\prime}$ form a basis of the dual cone $\check{\Gamma}$.

The family $u^{C_{1}}, \ldots, u^{C_{k}}$ generates the $\mathbf{C}$-algebra $A_{\Gamma}=\mathbf{C}\left[\check{\Gamma} \cap \mathbf{Z}^{k}\right]$. Here we write $u^{C_{j}}=u_{1}^{c_{1 j}} \cdots u_{k}^{c_{k j}}$ where the $c_{i j}$ 's are the entries of the column $C_{j}$.

We will write $W_{i}=U^{C_{i}}$ for $i=1, \ldots, k$. Similarly, we have $U_{j}=W^{C_{j}(\mathcal{L})}$ for $j=1, \ldots, k$ where $C_{j}(\mathcal{L})$ is the $j-t h$ column of the matrix $\mathcal{L}$. In Section 4 we will only use the column $C_{j}=C_{j}\left(\mathcal{L}^{\prime}\right)$.

Let us remark here that if $k<n$ then we can still define $W$, the relationship between $W$ and $U$ being exactly the same.

Proposition 2.8. The ring $\mathcal{R}^{\Gamma}(\mathcal{D})$ is isomorphic to the subring of $\mathcal{A}^{\prime}=$ $\mathbf{C}\{X, W\}[\Delta]$, consisting of all linear combinations with polynomials coefficients of convergent power series with respect to the family of monomials $X_{i} U_{i}=$ $X_{i} W^{C_{i}(\mathcal{L})}$ for $i=1, \ldots, k$, and $X_{k+1}, \ldots, X_{n}$.

Proof. A monomial $X^{\alpha} \Delta^{\beta} U^{s-\beta+\alpha}$ can be written in terms of $X, \Delta, W$ as $X^{\alpha} \Delta^{\beta} W^{\mathcal{L}(s-\beta+\alpha)}$. All that remains to be done is to enumerate the monomials derived from the power series variables $x_{1}, \ldots, x_{n}$

The ring $A_{\Gamma}$ is identified to the subring $\mathbf{C}[W]\left(=\mathbf{C}\left[W_{1}, \ldots, W_{k}\right]\right) \subset \mathcal{R}^{\Gamma}(\mathcal{D})$ and this inclusion is flat. We shall prove this statement in 4.2 .

The fiber of $\mathcal{R}^{\Gamma}(\mathcal{D})$ at the origin is by definition $\mathcal{R}^{\Gamma}(\mathcal{D}) \otimes_{\mathbf{C}[W]} \frac{\mathbf{C}[W]}{(W)}$ which is isomorphic to a Weyl algebra, namely the Weyl algebra $\mathbf{C}[X, \Delta]$. This Weyl algebra is endowed with a $\mathbf{Z}^{k}$-graduation by weight $\left(X_{i}\right)=-\epsilon_{i}$ and weight $\left(\Delta_{i}\right)=\epsilon_{i}$ for $i=1, \ldots, k$ where $\epsilon_{i}$ is the vector in $\mathbf{Z}^{k}$ whose $j-t h$ coordinate is $\delta_{i j}$.

\section{$\S 2.3$. Multifiltrations and Bernstein-Sato functional equations}

Let us detail the functional equation problem raised in the introduction. It has already been remarked that it gives rise to a situation where a multifiltration along transverse hypersurfaces comes out in a natural way. These equations are of the type

$$
P(\lambda) f^{\lambda}=b(\lambda) f_{1}^{\lambda_{1}+1} \cdots f_{k}^{\lambda_{k}+1}
$$

where $\lambda=\left(\lambda_{1}, \ldots, \lambda_{k}\right)$ is a $k$-uple of indeterminates, and $f=\left(f_{1}, \ldots, f_{k}\right)$ is a $k$-uple of analytic functions on $X$. They are naturally written in the module 
$\mathcal{O}_{X}\left[\lambda_{1}, \ldots, \lambda_{k}, \frac{1}{f_{1} \cdots f_{k}}\right] f^{\lambda}$, endowed with a structure of a $\mathcal{D}_{X}[\lambda]$-module, which can be extended in a way discovered by B. Malgrange to a $\mathcal{D}_{X \times \mathbf{C}^{k}}$-module structure with an action of the $2 k$ variables $t_{j}, \partial_{t_{j}}$ such that $\lambda_{j}=-\partial_{t_{j}} t_{j}$. The module $\mathcal{M}$ that we have then to consider is the module generated over the ring $\mathcal{D}_{X \times \mathbf{C}^{k}}$ by $f^{\lambda}$, with its naturally defined multifiltration $V_{\bullet}\left(\mathcal{D}_{X \times \mathbf{C}^{k}}\right) \cdot f^{\lambda}$ along the hypersurfaces $t_{1}=0, \cdots, t_{k}=0$. The proof of Sabbah in [9] can be sketched as follows: let us define for any linear form $L \in\left(\mathbf{Q}^{k}\right)^{\star}$ with positive rational coefficients $\ell_{j} \geq 0$, a filtration of $U_{\bullet}^{L}(\mathcal{M})$, associated with the linear combination $\sum \ell_{j} V^{(j)}$ of the basic $V$ - filtrations (see notations at 2). Precisely we set (see also Notation 3.1 below):

$$
\begin{gathered}
V_{\bullet}^{L}\left(\mathcal{D}_{X \times \mathbf{C}^{k}}\right)=\left\{P=\sum_{\mu, \nu \in \mathbf{N}^{k}} P_{\mu \nu}\left(x, \partial_{x}\right) t^{\mu} \partial_{t}^{\nu}, \sum \ell_{i}\left(\nu_{i}-\mu_{i}\right) \leq \bullet\right\} \\
U_{\bullet}^{L}(\mathcal{M})=V_{\bullet}^{L}\left(\mathcal{D}_{X \times \mathbf{C}^{k}}\right) \cdot f^{\lambda}
\end{gathered}
$$

Then the existence of a functional equation, in which $b(\lambda)$ is a product of affine forms $L(\lambda)+c$ comes out from the following three steps:

1) There are Bernstein-Sato polynomials $b_{L}(\lambda)$, relative to each $L$, with functional equations

$$
b_{L}(\lambda) f^{\lambda} \in V_{<0}^{L}\left(\mathcal{D}_{X \times \mathbf{C}^{k}}\right) \cdot f^{\lambda} .
$$

2) The saturated filtration

$$
\overline{U_{s}}(\mathcal{M})=\bigcap_{L \in\left(\mathbf{Q}^{k}\right)^{\star}} U_{L(s)}^{L}(\mathcal{M})
$$

can be defined by using only a finite number of fixed linear forms.

3) The saturated filtration is good which is equivalent to the existence of a $k$-uple of integers $\kappa$, such that

$$
\forall s \in \mathbf{N}^{k}, \quad U_{s}(\mathcal{M}) \subset \overline{U_{s}}(\mathcal{M}) \subset U_{s+\kappa}(\mathcal{M}) .
$$

It is step 2) which, in the proof in [9], makes an essential use of the notion of an adapted fan, whose existence is proved in this paper, see Theorem 2.6. In Bahloul's paper [4], this step is made by constructive methods which do not use the flatness property. 


\section{$\S 3 . \quad$ The Analytic Standard Fan}

In this section we will summarize the main results of [3]. Since these results are only given for a module $\mathcal{D} / I$ over $\mathcal{D}$ which is a quotient by an ideal we will then show briefly how to adapt them to a module of the type $\frac{\mathcal{D}^{r}}{\mathcal{N}}$. Let us remark that the fan used in this paper is obtained by a restriction of the fan in [3] to a subset of linear forms of the set $\mathcal{U}$ defined below, namely the linear combinations of the filtrations $V^{(i)}$.

Let $\mathcal{U}$ be the set of linear forms $\Lambda: \mathbf{R}^{2 n} \rightarrow \mathbf{R}, \Lambda(\alpha, \beta)=\sum_{i=1}^{n} e_{i} \alpha_{i}+$ $\sum_{i=1}^{n} f_{i} \beta_{i}$ with $e_{i}+f_{i} \geq 0$ and $e_{i} \leq 0$ for $i=1, \ldots, n$. If

$$
P=\sum_{\alpha \beta} p_{\alpha \beta} x^{\alpha} \partial^{\beta}
$$

is an element in $\mathcal{D}$ we define $\operatorname{ord}^{\Lambda}(P)$-the $\Lambda$-order of $P$ - to be the maximal value of $\Lambda(\alpha, \beta)$ for $\alpha, \beta$ such that $p_{\alpha \beta} \neq 0$.

The $\Lambda$-filtration $F_{\Lambda, \bullet}(\mathcal{D})$ is defined by

$$
F_{\Lambda, \ell}=F_{\Lambda, \ell}(\mathcal{D})=\left\{P \in \mathcal{D} \mid \operatorname{ord}^{\Lambda}(P) \leq \ell\right\}
$$

for any $\ell \in \mathbf{R}$. We will write $F_{\Lambda,<\ell}(\mathcal{D}):=\left\{P \in \mathcal{D} \mid \operatorname{ord}^{\Lambda}(P)<\ell\right\}$. If $e_{i}=$ $0, f_{i}=1$ for $i=1, \ldots, n$ the corresponding $\Lambda$-filtration is nothing but the usual filtration by the order of differential operators. We shall denote it simply $F_{\bullet}(\mathcal{D})$.

Let us recall that we have fixed $k \leq n$. For each linear form $L \in\left(\mathbf{Q}^{k}\right)_{+}^{*}$ (i.e. the coefficients of $L$ are non-negative) we denote by $\widetilde{L}$ the linear form on $\mathbf{R}^{2 n}$ defined by $\widetilde{L}(\alpha, \beta)=L(\beta)-L(\alpha)$ where $L\left(\alpha_{1}, \ldots, \alpha_{n}\right)=L\left(\alpha_{1}, \ldots, \alpha_{k}\right)$.

Notation 3.1. We define the filtration $V_{\bullet}^{L}$ on $\mathcal{D}$, indexed by the set of values $L\left(\mathbf{Z}^{k}\right)$, as:

$$
V_{\ell}^{L}(\mathcal{D})=F_{\widetilde{L}, \ell}(\mathcal{D})=\left\{P \in \mathcal{D} \mid \operatorname{ord}^{\widetilde{L}}(P) \leq \ell\right\}
$$

Let us fix $L \in\left(\mathbf{Q}^{k}\right)_{+}^{*}$. The graded ring associated to the filtration $V^{L}=F_{\widetilde{L}}$ on $\mathcal{D}$, is by definition

$$
\operatorname{gr}^{\widetilde{L}}(\mathcal{D})=\bigoplus_{\ell \in \widetilde{L}\left(\mathbf{Z}^{2 n}\right)} \frac{F_{\widetilde{L}, \ell}}{F_{\widetilde{L},<\ell}} .
$$

If no confusion is possible we shall write simply $\operatorname{ord}^{L}$ and $\operatorname{gr}^{L}(\mathcal{D})$ instead of $\operatorname{ord}^{\widetilde{L}}$ and $\operatorname{gr}^{\widetilde{L}}(\mathcal{D})$.

The graded ring $\operatorname{gr}^{L}(\mathcal{D})$ is a ring of differential operators and its structure is the following: suppose the coefficients of the form $L$ are $\left(e_{1}, \ldots, e_{k}\right)$ and 
suppose we also have ordered the variables to have $e_{i}>0$ for $1 \leq i \leq \ell$ for some $\ell \leq k$.

Then the graded $\operatorname{ring} \operatorname{gr}^{L}(\mathcal{D})$ is isomorphic to the ring

$$
\mathbf{C}\left\{x_{\ell+1}, \ldots, x_{n}\right\}\left[x_{1}, \ldots, x_{\ell}, \partial_{1}, \ldots, \partial_{n}\right] .
$$

In this ring the graduation is induced by the weights weight $\left(x_{i}\right)=-e_{i}$, weight $\left(\partial_{i}\right)=e_{i}$ for $1 \leq i \leq \ell$ and $\operatorname{weight}\left(x_{j}\right)=\operatorname{weight}\left(\partial_{j}\right)=0$ otherwise. There is only a finite number of types of these rings, one for each partition of $\{1, \ldots, k\}$ into two sets.

Notation 3.2. For each $P \in \mathcal{D}$ and for each $d \in L\left(\mathbf{Z}^{k}\right)$ with $\operatorname{ord}^{L}(P) \leq$ $d$ we denote by $\sigma_{d}^{L}(P)$-the $L$-symbol of $P$ of order $d$-the class of $P$ in $F_{\widetilde{L}, d} /$ $F_{\widetilde{L},<d}$. The principal symbol of $P$ is by definition $\sigma^{L}(P)=\sigma_{d}^{L}(P)$ if $d=$ $\operatorname{ord}^{L}(P)$. For $P, Q \in \mathcal{D}$ we have $\sigma^{L}(P Q)=\sigma^{L}(P) \sigma^{L}(Q)$. For any left ideal $I$ in $\mathcal{D}$ we denote by $\operatorname{gr}^{L}(I)$ the graded ideal of $\operatorname{gr}^{L}(\mathcal{D})$ generated by the set $\left\{\sigma^{L}(P) \mid P \in I\right\}$. We set $\sigma^{L}(0)=0$.

We denote by $\mathcal{D}[t]$ the $\mathbf{C}$-algebra $\mathcal{O}[\partial, t]=\mathbf{C}\left\{x_{1}, \ldots, x_{n}\right\}\left[\partial_{1}, \ldots, \partial_{n}, t\right]$ with relations ( $t$ being a new variable)

- $[t, a]=\left[t, \partial_{i}\right]=[a, b]=\left[\partial_{i}, \partial_{j}\right]=0$

- $\left[\partial_{i}, a\right]=\frac{\partial a}{\partial x_{i}} t$,

for $a, b \in \mathcal{O}$ and $i=1, \ldots, n$.

The ring $\mathcal{D}[t]$ is isomorphic to the Rees ring associated with the order filtration $F_{\bullet}$ on $\mathcal{D}$. Since this Rees ring is by definition

$$
\mathcal{R}_{F}(\mathcal{D})=\bigoplus_{\ell \in \mathbf{Z}} F_{\ell}(\mathcal{D}) v^{\ell} \subset \mathcal{D}[v]=\mathcal{D} \otimes_{\mathbf{C}} \mathbf{C}[v]
$$

for a new variable $v$, we can define an isomorphism of graded rings $\iota: \mathcal{D}[t] \rightarrow$ $\mathcal{R}_{F}(\mathcal{D})$ by $\iota(a)=a=a v^{0}, \iota\left(\partial_{j}\right)=\partial_{j} v$, and $\iota(t)=1 v=v$.

The natural graded structure of $\mathcal{R}_{F}(\mathcal{D})$ can be translated on $\mathcal{D}[t]$. An homogeneous element of degree $d \in \mathbf{Z}$ in $\mathcal{D}[t]$ is nothing but an expression

$$
\sum_{\ell+|\beta|=d} a_{\ell \beta} \partial^{\beta} t^{\ell}
$$

for some $a_{\ell \beta} \in \mathcal{O}$.

For $P=\sum_{\beta} p_{\beta}(x) \partial^{\beta} \in \mathcal{D}$, the element $P v^{\operatorname{ord}(P)} \in \mathcal{R}_{F}(\mathcal{D})$ is called the homogenization of $P$, where $\operatorname{ord}(P)$ is the usual order of $P$. It is useful to see 
$P v^{\operatorname{ord}(P)}$ as an element of $\mathcal{D}[t]$. The homogenization of $P$ is then denoted by $h(P)$ and we have

$$
h(P)=\sum_{\beta} p_{\beta}(x) \partial^{\beta} t^{d-|\beta|}
$$

for $d=\operatorname{ord}(P)$ the usual order of $P$.

For each linear form $L \in\left(\mathbf{Q}^{k}\right)_{+}^{*}$ we can define in a natural way a filtration $V_{\bullet}^{L}(\mathcal{D}[t])$ on $\mathcal{D}[t]$, the $L$-order -denoted $\operatorname{ord}^{L}(R)$ - of an element

$$
R=\sum_{\ell \alpha \beta} r_{\ell \alpha \beta} x^{\alpha} \partial^{\beta} t^{\ell}
$$

being the maximal value of $L(\beta)-L(\alpha)$ for $r_{\ell \alpha \beta} \neq 0$. The associated graded ring is denoted by $\operatorname{gr}^{L}(\mathcal{D}[t])$. We have a natural ring isomorphism from $\operatorname{gr}^{L}(\mathcal{D}[t])$ onto $\operatorname{gr}^{L}(\mathcal{D})[t]$, where with the notations as before we have $\left[\partial_{i}, x_{j}\right]=\delta_{i j} t, \delta_{i j}$ being the Kronecker symbol.

For each left ideal $I$ in $\mathcal{D}$ we denote by $h(I)$ the left homogeneous ideal of $\mathcal{D}[t]$ generated by $\{h(P) \mid P \in I\}$. As in the case of $\mathcal{D}$, we denote by $\operatorname{gr}^{L}(h(I))$ the homogeneous ideal -in $\operatorname{gr}^{L}(\mathcal{D}[t])$ - generated by the set of principal symbols $\sigma^{L}(G)$ of the elements $G$ in $h(I)$.

The main result of [3] is the following

Theorem 3.3. $\quad$ Let $I$ be a non-zero left ideal of $\mathcal{D}$ and let $h(I)$ be the associated homogenized ideal in $\mathcal{D}[t]$. Then there exists a partition $\mathcal{E}$ of $\mathcal{U}$ into convex rational polyhedral cones such that for any $\Gamma \in \mathcal{E}$ the ideals $\operatorname{gr}^{\Lambda}(h(I))$ and $\operatorname{gr}^{\Lambda}(I)$ do not depend on $\Lambda \in \Gamma$.

Let us sketch the generalization of this result to the case of a $\mathcal{D}$-module $\mathcal{M}=\frac{\mathcal{D}^{r}}{\mathcal{N}}$ given by a general presentation, and endowed with a good multifiltration

$$
U_{s}(\mathcal{M})=\frac{V_{s}[\underline{n}]\left(\mathcal{D}^{r}\right)}{V_{s}(\mathcal{N})}=\frac{V_{s}[\underline{n}]\left(\mathcal{D}^{r}\right)+\mathcal{N}}{\mathcal{N}}
$$

associated with a multivector shift $\underline{n} \in\left(\mathbf{Z}^{k}\right)^{r}$.

Here we have to restrict the set $\mathcal{U}$ to the set of linear forms $\widetilde{L}$, indexed by $L$ in $\left(\mathbf{Q}^{k}\right)_{+}^{*}$, as defined above:

$$
\widetilde{L}(\alpha, \beta)=L(\beta)-L(\alpha) .
$$

Let us recall Notation $3.1 V_{L(s)}^{L}(\mathcal{D})=F_{\widetilde{L}, L(s)}(\mathcal{D})$, which we shall extend to any free multifiltered module.

Precisely, given a shift multivector $\underline{n}=\left(n^{(1)}, \ldots, n^{(r)}\right) \in\left(\mathbf{Z}^{k}\right)^{r}$ we define a family, indexed by $L \in\left(\mathbf{Q}^{k}\right)_{+}^{*}$, of $L\left(\mathbf{Z}^{k}\right)$-filtrations on the module $\mathcal{M}$ as follows: 


$$
V_{\ell}^{L}[L(\underline{n})]\left(\mathcal{D}^{r}\right)=\bigoplus_{i=1}^{r} V_{\ell-\ell_{i}}^{L}(\mathcal{D})
$$

where $\ell_{i}=L\left(n^{(i)}\right)$ for $i=1, \ldots, r$ and $L(\underline{n})=\left(\ell_{1}, \ldots, \ell_{r}\right)$.

$$
U_{\ell}^{L}(\mathcal{M})=\frac{V_{\ell}^{L}[L(\underline{n})]\left(\mathcal{D}^{r}\right)}{V_{\ell}^{L}(\mathcal{N})}=\frac{V_{\ell}^{L}[L(\underline{n})]\left(\mathcal{D}^{r}\right)+\mathcal{N}}{\mathcal{N}} .
$$

We again have the notion of a graded associated module

$$
\operatorname{gr}^{L}(\mathcal{M})=\bigoplus_{\ell \in L\left(\mathbf{Z}^{k}\right)} \frac{U_{\ell}^{L}(\mathcal{M})}{U_{<\ell}^{L}(\mathcal{M})}=\frac{\operatorname{gr}^{L}\left(\mathcal{D}^{r}\right)}{\operatorname{gr}^{L}(\mathcal{N})} .
$$

We can endow the module $\mathcal{D}[t]^{r}$ with a filtration of a filtered $\mathcal{D}[t]$-module by

$$
V_{\ell}^{L}[L(\underline{n})]\left(\mathcal{D}[t]^{r}\right)=\bigoplus_{i=1}^{r} V_{\ell-\ell_{i}}^{L}(\mathcal{D}[t])
$$

with the same notations as before, and the homogeneization of $\left(P_{1}, \ldots, P_{r}\right) \in$ $\mathcal{D}^{r}$ is just

$$
\left(t^{d-d_{1}} h\left(P_{1}\right), \ldots, t^{d-d_{r}} h\left(P_{r}\right)\right)
$$

where $d_{i}$ is the usual order of the operator $P_{i}$ and $d=\max d_{i}$.

We can now state the straightforward generalization of the main theorem in [3].

Theorem 3.4. $\quad$ Let $\mathcal{N}$ be a non-zero left submodule of $\mathcal{D}^{r}$ endowed with a good filtration, and let $h(\mathcal{N})$ be the associated homogenized submodule in $\mathcal{D}[t]^{r}$. Then there exists a partition $\mathcal{E}$ of $\left(\mathbf{Q}^{k}\right)_{+}^{*}$ into convex rational polyhedral cones such that for any $\Gamma \in \mathcal{E}$, the submodules $\operatorname{gr}^{L}(h(\mathcal{N}))$ and $\operatorname{gr}^{L}(\mathcal{N})$ do not depend on $L \in \Gamma$.

The proof of the theorem is identical to the one in [3], since the main ingredient, which is the division theorem, can be adapted to the module case. See for example [6], where an even more general situation (with shifts for the variables $t$ ) is treated. In particular we still have on every cone of the partition $\mathcal{E}$ the notion of a reduced Gröbner basis of $h(\mathcal{N})$, valid for any linear form $L$ in the cone. 


\section{$\S 4 . \quad$ Analytic Standard Fan and Flatness}

After Herrmann-Orbanz [7] we have the following criterion for flatness of a module $M$ over a commutative ring $R$, both being graded by a commutative group $G$ :

$$
M=\bigoplus_{g \in G} M_{g}, \quad R=\bigoplus_{g \in G} R_{g}
$$

Theorem 4.1. The (not necessarily of finite type) graded $R$-module $M$ is $R$-flat if and only if for any graded ideal $H \subset R$, we have:

$$
\operatorname{Tor}_{1}^{R}(M, R / H)=0 .
$$

We will apply this criterion to the Rees module

$$
\mathcal{R}^{\Gamma}(\mathcal{M})=\bigoplus_{s \in \mathbf{Z}^{k}} U_{s}^{\Gamma}(\mathcal{M}) u^{s}
$$

graded by the group $\mathbf{Z}^{k}$, seen as a module over the graded ring $\mathcal{R}^{\Gamma}(\mathcal{D})$, and therefore also as a module over the graded subring $R=\mathbf{C}[W]=\mathbf{C}\left[W_{1}, \ldots, W_{k}\right]$ (see 2.2).

Note that for any $a \in \mathbf{N}^{k}, \mathbf{C} \cdot W^{a}$, is a homogeneous subspace of $R$ generated by a non zero element of $\mathbf{Z}^{k}$-degree $\mathcal{L}^{\prime} a$, since with the notation of Section 2.2.3 $W^{a}=u^{\mathcal{L}^{\prime} a}$. Therefore the graded ideals of $R$ we have to take into consideration are the monomial ideals of the polynomial ring $R$.

We now deal with the case of $\mathcal{M}=\frac{\mathcal{D}^{r}}{\mathcal{N}}$. If we endow $\mathcal{N}$ and $\mathcal{M}$ with the induced and quotient filtration, from a $V \bullet[\underline{n}]$-filtration on the free module $\mathcal{D}^{r}$, we have the exact sequence:

$$
0 \longrightarrow \mathcal{R}^{\Gamma}(\mathcal{N}) \longrightarrow \mathcal{R}^{\Gamma}\left(\mathcal{D}^{r}\right) \longrightarrow \mathcal{R}^{\Gamma}(\mathcal{M}) \longrightarrow 0 .
$$

Lemma 4.2. The ring $\mathcal{R}^{\Gamma}(\mathcal{D})$ is flat over $\mathbf{C}[W]$.

Proof. By the theorem of Herrmann-Orbanz, it is sufficient to prove that

$$
\operatorname{Tor}_{1}^{\mathbf{C}[W]}\left(\mathcal{R}^{\Gamma}(\mathcal{D}), \mathbf{C}[W] / H\right)=0
$$

for any homogeneous ideal $H$. If we consider the tensor product by $\mathcal{R}^{\Gamma}(\mathcal{D})$ of the exact sequence

$$
0 \longrightarrow H \longrightarrow \mathbf{C}[W] \longrightarrow \mathbf{C}[W] / H \longrightarrow 0
$$


we see that this vanishing result is equivalent to the injectivity of the mapping

$$
H \otimes \mathcal{R}^{\Gamma}(\mathcal{D}) \longrightarrow \mathcal{R}^{\Gamma}(\mathcal{D}) .
$$

Since the ideal $H$ is multihomogeneous, it is a monomial ideal and we may consider an element $\mathfrak{Q}=W^{a_{1}} \otimes Q_{1}+\cdots+W^{a_{r}} \otimes Q_{r}$ of the kernel of this map. Let $Q_{i}=\sum q_{i, \alpha, \beta, \ell} X^{\alpha} \Delta^{\beta} W^{\ell}$ be the development of $Q_{i}$ in the ring $\mathcal{R}^{\Gamma}(\mathcal{D})$ identified to the subring of $\mathcal{A}^{\prime}$ described in Proposition 2.8.

The fact that $\mathfrak{Q}$ is in the kernel can be expressed by the equation

$$
\sum_{i=1}^{r} W^{a_{i}} Q_{i}=0
$$

Let us consider the partition of $\bigcup\left(a_{i}+\mathbf{N}^{k}\right) \subset \mathbf{N}^{k}$ given by:

$$
\Delta_{1}=a_{1}+\mathbf{N}^{k}, \quad \Delta_{i}=\left(a_{i}+\mathbf{N}^{k}\right) \backslash\left(\Delta_{1} \cup \cdots \cup \Delta_{i-1}\right), \text { for } i=2, \ldots, r
$$

We may write $Q_{i}=\sum_{p=1}^{i} Q_{i, p}$, with:

$$
Q_{i, p}=\sum_{\ell+a_{i} \in \Delta_{p}} q_{i, \alpha, \beta, \ell} X^{\alpha} \Delta^{\beta} W^{\ell}
$$

operators having disjoint ( $W$ )-Newton diagrams. We set $a_{i}+v_{i, p}=a_{p}+w_{i, p}$, with $v_{i, p}, w_{i, p} \in \mathbf{N}^{k}$ minimal for the componentwise partial ordering, and then we may write $Q_{i, p}=W^{v_{i, p}} R_{i, p}$ with $R_{i, p} \in \mathcal{D}$, and we may also remark that $W^{a_{i}} Q_{i, p}$ is the part of the operator $W^{a_{i}} Q_{i}$, whose Newton diagram is contained in $\Delta_{p}$. According to the description in the Proposition 2.8, this is sufficient to guarantee that $Q_{i, p}, R_{i, p} \in \mathcal{R}^{\Gamma}(\mathcal{D}) \subset \mathcal{A}^{\prime}$ so that the equation $(\star)$ splits inside the ring $\mathcal{R}^{\Gamma}(\mathcal{D})$ into:

$$
\sum_{i=p}^{r} W^{a_{i}} Q_{i, p}=W^{a_{p}} \sum_{i=p}^{r} W^{w_{i, p}} R_{i, p}=0 \text { for } p=1, \ldots, r .
$$

This finally implies the desired result by the following calculation:

$$
\begin{aligned}
\mathfrak{Q} & =\sum_{i=1}^{r} W^{a_{i}} \otimes\left(\sum_{p=1}^{i} W^{v_{i, p}} R_{i, p}\right)=\sum_{p=1}^{r} \sum_{i=p}^{r} W^{a_{i}+v_{i, p}} \otimes R_{i, p} \\
& =\sum_{p=1}^{r} \sum_{i=p}^{r} W^{a_{p}+w_{i, p}} \otimes R_{i, p}=\sum_{p=1}^{r} W^{a_{p}} \otimes\left(\sum_{i=p}^{r} W^{w_{i, p}} R_{i, p}\right)=0
\end{aligned}
$$


By the theorem of Herrmann-Orbanz, the flatness of $\mathcal{R}^{\Gamma}(\mathcal{M})$ - if $\Gamma$ is included in a cone of the analytic fan of $h(\mathcal{N})$ - is a consequence of the equality

$$
\operatorname{Tor}_{1}^{\mathbf{C}[W]}\left(\mathcal{R}^{\Gamma}(\mathcal{M}), \mathbf{C}[W] / H\right)=0
$$

for any homogeneous, that is monomial, ideal $H \subset \mathbf{C}[W]$.

Because of the above lemma, and of the exact sequence $(\mathcal{S})$, this Tor module appears as the kernel of the map:

$$
\mathbf{C}[W] / H \otimes_{\mathbf{C}[W]} \mathcal{R}^{\Gamma}(\mathcal{N})=\frac{\mathcal{R}^{\Gamma}(\mathcal{N})}{H \cdot \mathcal{R}^{\Gamma}(\mathcal{N})} \longrightarrow \frac{\mathcal{R}^{\Gamma}\left(\mathcal{D}^{r}\right)}{H \cdot \mathcal{R}^{\Gamma}\left(\mathcal{D}^{r}\right)}
$$

and we will prove that it is injective.

The injectivity means that $H \cdot \mathcal{R}^{\Gamma}(\mathcal{N})=H \cdot \mathcal{R}^{\Gamma}\left(\mathcal{D}^{r}\right) \cap \mathcal{R}^{\Gamma}(\mathcal{N})$ for any monomial ideal $H=\sum_{i=1}^{\ell} \mathbf{C}[W] W^{a_{i}}$. The inclusion $H \cdot \mathcal{R}^{\Gamma}(\mathcal{N}) \subset H \cdot \mathcal{R}^{\Gamma}\left(\mathcal{D}^{r}\right) \cap$ $\mathcal{R}^{\Gamma}(\mathcal{N})$ is obvious.

Theorem 4.3. Let $\Gamma$ be a cone included in the closure of a cone of the analytic standard fan of $h(\mathcal{N})$. Then $\mathcal{R}^{\Gamma}(\mathcal{M})$ is flat over $A_{\Gamma}=\mathbf{C}[W]$.

Proof. We have to prove the inclusion $H \cdot \mathcal{R}^{\Gamma}(\mathcal{N}) \supset H \cdot \mathcal{R}^{\Gamma}\left(\mathcal{D}^{r}\right) \cap \mathcal{R}^{\Gamma}(\mathcal{N})$ for any monomial ideal $H$ in $\mathbf{C}[W]$. It is enough to treat the case where $H$ is generated by a set of variables $\left\{W_{j_{1}}, \ldots, W_{j_{p}}\right\}$ for some $J=\left\{j_{1}, \ldots, j_{p}\right\} \subset$ $\{1, \ldots, k\}$. We denote by $W_{J}$ the ideal generated by $\left\{W_{j_{1}}, \ldots, W_{j_{p}}\right\}$ and we call it a coordinate ideal. This is due to the two following lemmata.

Lemma 4.4. Let $H_{1} \subset H_{2} \subset \mathbf{C}[W]$ be two ideals and $M a \mathbf{C}[W]$ module such that

$$
\operatorname{Tor}_{1}^{\mathbf{C}[W]}\left(M, \mathbf{C}[W] / H_{2}\right)=\operatorname{Tor}_{1}^{\mathbf{C}[W]}\left(M, H_{2} / H_{1}\right)=0
$$

then $\operatorname{Tor}_{1}^{\mathbf{C}[W]}\left(M, \mathbf{C}[W] / H_{1}\right)=0$.

Lemma 4.5. Let $H$ be a monomial ideal in $\mathbf{C}[W]=\mathbf{C}\left[W_{1}, \ldots, W_{k}\right]$. Then there exists a sequence of monomial ideals

$$
H=H_{0} \subset H_{1} \subset \cdots \subset H_{r}=\mathbf{C}[W]
$$

such that for any $i$ the quotient $H_{i+1} / H_{i}$ is isomorphic to $\mathbf{C}[W] / W_{J(i)}$ for some $J(i) \subset\{1, \ldots, k\}$.

Proof. By noetherianity of $\mathbf{C}[W]$ it is sufficient to construct $H_{1}=H_{0}+$ $\mathbf{C}[W] m$ where $m$ is a monomial in $\mathbf{C}[W]$ such that $m \notin H_{0}$ and $\left(H_{0}: m\right)$ is 
some coordinate ideal $W_{J}$. In the artinian case just take an element in the socle and in general use an induction on the dimension of $\mathbf{C}[W] / H$.

So, in order to prove Theorem 4.3 it is sufficient to treat the case $H$ generated by $\left\{W_{1}, \ldots, W_{p}\right\}$ for any $p \leq k$. We have (see 2.2.3) $W_{i}=U^{C_{i}}$ for $i=1, \ldots, k$ where $C_{i} \in \mathbf{Z}^{k}$ is the $i$-th column of the matrix $\mathcal{L}^{\prime}$. Since the considered submodules of $\mathcal{R}^{\Gamma}\left(\mathcal{D}^{r}\right)$ are $V_{\bullet}[\underline{n}]$-homogeneous, it is sufficient to prove that any homogeneous element $Q u^{s} \in H \cdot \mathcal{R}^{\Gamma}\left(\mathcal{D}^{r}\right) \cap \mathcal{R}^{\Gamma}(\mathcal{N})$ belongs to $H \cdot \mathcal{R}^{\Gamma}(\mathcal{N})$. As $H \cdot \mathcal{R}^{\Gamma}\left(\mathcal{D}^{r}\right)$ is the submodule $\sum u^{C_{i}} \mathcal{R}^{\Gamma}\left(\mathcal{D}^{r}\right)$, the vector-operator $Q$, which belongs to $\mathcal{N}$, can be written $Q=\sum_{i=1}^{p} Q_{i}$ for some $Q_{i} \in V_{s-C_{i}}^{\Gamma}\left(\mathcal{D}^{r}\right)$.

For any $i, j=1, \ldots, p$ we have (see Notation 3.2):

$$
\begin{gathered}
\operatorname{ord}^{L_{i}}\left(Q_{j}\right) \leq L_{i}(s)-\delta_{i j} . \\
\sigma_{L_{i}(s)}^{L_{i}}\left(\sum_{j} Q_{j}\right)=\sigma_{L_{i}(s)}^{L_{i}}\left(\sum_{j \neq i} Q_{j}\right) .
\end{gathered}
$$

There exists a family $\ell, \ell_{1}, \ldots, \ell_{p}$ of non-negative integer numbers such that

$$
t^{\ell} h(Q)=\sum_{i} t^{\ell_{i}} h\left(Q_{i}\right)
$$

Let us consider $H_{1}, \ldots, H_{q}$ a simultaneous reduced $L$-standard basis of $h(\mathcal{N})$ for any $L$ in $\Gamma$. The existence of such a simultaneous $L$-standard basis is a consequence of the inclusion of $\Gamma$ in the closure of a cone of the analytic standard fan of $h(\mathcal{N})$ (see the proof of [3, Th. 20]). By the analytic division theorem in $\mathcal{D}[t]$ (see $[3$, Th. 7$]$, and $[6$, Th. 4.1.]) we can write

$$
t^{\ell} h(Q)=\sum_{m=1}^{q} A_{m} H_{m}
$$

for some $F$-homogeneous elements $A_{j} \in \mathcal{D}[t]$.

For an element $B=\sum_{\alpha \beta i} b_{\alpha \beta i} x^{\alpha} \partial^{\beta} e_{i}$ in $\mathcal{D}^{r}$ we denote by $\mathcal{N}_{V}(B)$ its $V_{\bullet}[\underline{n}]-$ Newton diagram. By definition this is the subset of $\mathbf{Z}^{k}$ defined by:

$$
\mathcal{N}_{V}(B)=\left\{\left(\beta_{1}-\alpha_{1}+n_{1}^{(i)}, \ldots, \beta_{k}-\alpha_{k}+n_{k}^{(i)}\right) \in \mathbf{Z}^{k} \mid b_{\alpha \beta i} \neq 0\right\} .
$$

If no confusion is possible we shall denote $\beta-\alpha+n^{(i)}=\left(\beta_{1}-\alpha_{1}+n_{1}^{(i)}, \ldots, \beta_{k}-\right.$ $\left.\alpha_{k}+n_{k}^{(i)}\right)$. We have an analogous definition for the $V \cdot[\underline{n}]$-Newton diagram of an element in $\mathcal{D}[t]^{r}$.

We have

$$
\mathcal{N}_{V}\left(t^{\ell} h(Q)\right)=\mathcal{N}_{V}(Q) \subset \bigcup_{i=1}^{p}\left(\left(s-C_{i}\right)-\check{\Gamma}\right) .
$$


Since the operators $H_{m}$ are elements of an $L$-standard basis for any $L \in \Gamma$ we have $\mathcal{N}_{V}\left(H_{m}\right) \subset \exp \left(H_{m}\right)-\check{\Gamma}$, and therefore as a consequence of the division theorem in $\mathcal{D}[t]$, we have $\mathcal{N}\left(A_{m}\right)+V_{\bullet}[\underline{n}]\left(\exp \left(H_{m}\right)\right) \subset \bigcup_{i=1}^{p}\left(\left(s-C_{i}\right)-\check{\Gamma}\right)$ for $m=1, \ldots, q$, where $V_{\bullet}[\underline{n}](\alpha, \beta, i)$ if by definition $\beta-\alpha+n^{(i)}$ and exp stands for the privileged exponent with respect to a previously fixed well ordering on $\mathbf{N}^{2 n+1} \times\{1 \cdots r\}$ (see [6, pp. 174-175] for more details).

As a consequence of the division theorem we also have

$$
\begin{aligned}
\sigma_{L_{1}(s)}^{L_{1}}\left(t^{\ell} h(Q)\right) & =\sum_{j=2}^{p} \sigma_{L_{1}(s)}^{L_{1}}\left(t^{\ell_{j}} h\left(Q_{j}\right)\right) \\
& =\sum_{m=1}^{q} \sigma_{L_{1}(s)}^{L_{1}}\left(A_{m} H_{m}\right)=\sum_{m=1}^{q} a_{m}(x, \partial, t) \sigma_{d_{1, m}}^{L_{1}}\left(H_{m}\right)
\end{aligned}
$$

where $d_{1, m}=\operatorname{ord}^{L_{1}}\left(H_{m}\right)$ and $a_{m}(x, \partial, t)$ is $L_{1}$-homogeneous. As the $V_{\bullet}[\underline{n}]$ Newton diagram of $\sigma_{L_{1}(s)}^{L_{1}}\left(t^{\ell} h(Q)\right)=\sum_{j=2}^{p} \sigma_{L_{1}(s)}^{L_{1}}\left(t^{\ell_{j}} h\left(Q_{j}\right)\right)$ is included in $\bigcup_{j=2}^{p}\left(\left(s-C_{j}\right)-\check{\Gamma}\right)$ we have, for any $m=1, \ldots, q$ the inclusion

$$
\mathcal{N}_{V}\left(a_{m}(x, \partial, t)\right)+V_{\bullet}[\underline{n}]\left(\exp \left(H_{m}\right)\right) \subset \bigcup_{j=2}^{p}\left(\left(s-C_{j}\right)-\check{\Gamma}\right) .
$$

Let us write

$$
R_{1}=t^{\ell} h(Q)-\sum_{m=1}^{q} a_{m}(x, \partial, t) H_{m}, \text { and } Q_{1}^{\prime}=\left(R_{1}\right)_{\mid t=1} .
$$

We have:

$$
\begin{gathered}
\operatorname{ord}^{L_{1}}\left(R_{1}\right) \leq L_{1}(s)-1, \\
\operatorname{ord}^{L_{j}}\left(R_{1}\right) \leq L_{j}(s), j=2, \ldots, p,
\end{gathered}
$$

and we can decompose each $a_{m}(x, \partial, t)$ into a sum

$$
a_{m}(x, \partial, t)=\sum_{j=2}^{p} A_{m j}
$$

such that $\mathcal{N}_{V}\left(A_{m j}\right)+V_{\bullet}[\underline{n}]\left(\exp \left(H_{m}\right)\right) \subset\left(\left(s-C_{j}\right)-\check{\Gamma}\right)$ for $m=1, \ldots, q$ and $j=2, \ldots, p$.

Let us write $R_{j}=\sum_{m=1}^{q} A_{m j} H_{m} \in h(\mathcal{N})$ and $Q_{j}^{\prime}=\left(R_{j}\right)_{\mid t=1} \in \mathcal{N}$ for $j=2, \ldots, p$. 
We see $Q_{1}^{\prime}=Q-\sum_{j=2}^{p} Q_{j}^{\prime} \in \mathcal{N}$. We have $Q_{1}^{\prime} \in V_{s-C_{1}}^{\Gamma}(\mathcal{N})$ by the properties of the orders $\operatorname{ord}^{L_{j}}\left(R_{1}\right)$, and for $i=2, \ldots, k$ we have $Q_{i}^{\prime} \in V_{s-C_{i}}^{\Gamma}(\mathcal{N})$, by the properties of the $V_{\bullet}[\underline{n}]$-Newton diagram of the $A_{m j}$. Therefore we can write

$$
Q u^{s}=\left(R_{1}\right)_{\mid t=1}=Q_{1}^{\prime} u^{s-C_{1}} u^{C_{1}}+\cdots+Q_{p}^{\prime} u^{s-C_{p}} u^{C_{p}} \in H \mathcal{R}^{\Gamma}(\mathcal{N}) .
$$

That ends the proof.

\section{Acknowledgement}

During the preparation of this paper both authors have been partially supported by Acción Integrada-Picasso HF2004-0117. First author has been also partially supported by MTM2004-01165. Both authors are grateful to the Département de Mathématiques et Applications of the École Normale Supérieure (Paris) where the first was invited during the preparation of the final part of this work. They also thank Jenifer Granger for her proofreading.

\section{References}

[1] A. Assi, F. J. Castro-Jiménez and M. Granger, How to calculate the slopes of a $\mathcal{D}$ module, Compositio Math. 104 (1996), no. 2, 107-123.

[2] $\_$, The Gröbner fan of an $A_{n}$-module, J. Pure Appl. Algebra 150 (2000), no. 1, $27-39$.

[3] no. $1-2,3-21$

[4] R. Bahloul, Démonstration constructive de l'existence de polynômes de Bernstein-Sato pour plusieurs fonctions analytiques, Compos. Math. 141 (2005), no. 1, 175-191.

[5] R. Bahloul and N. Takayama, Local Gröbner fan: polyhedral and computational approach, Preprint arXiv:math.AG/0412044, December 2004.

[6] M. Granger and T. Oaku, Minimal filtered free resolutions for analytic $\mathcal{D}$-modules, J. Pure Appl. Algebra 191 (2004), no. 1-2, 157-180.

[7] M. Herrmann and U. Orbanz, Two notes on flatness, Manuscripta Math. 40 (1982), no. $1,109-133$

[8] C. Sabbah and F. Castro, Appendice à "proximité evanescente" I. La structure polaire d'un $\mathcal{D}$-module, Compositio Math. 62 (1987), no. 3, 320-328.

[9] C. Sabbah, Proximité évanescente. I. La structure polaire d'un $\mathcal{D}$-module, Compositio Math. 62 (1987), no. 3, 283-319. 